\title{
Assisting Text Annotation based on Attributes Suggestion Strategy using Content and Query
}

\author{
Vikas R. Palekar ${ }^{1}$, Miss. Shital P. Dhok ${ }^{2}$, Prof. Dinesh D. Gawande ${ }^{3}$ \\ Asst. Professor, Department of CSE, DMIETR, Sawangi (Wardha), Maharashtra (India) ${ }^{1}$ \\ Department of CSE, BDCOE, SEWAGRAM (Wardha), Maharashtra (India) ${ }^{2}$ \\ Asst. Professor, Department of CSE, DBACER, Nagpur, Maharashtra (India) ${ }^{3}$
}

\begin{abstract}
In the current computing world, machine based data innovations have been broadly used to help numerous associations, individual businesses, scholarly and training establishments to deal with their procedures and data frameworks. Data frameworks are utilized to keep an eye on information. A general information administration framework that is prepared to do dealing with a few sorts of information, data stored in the database is known as Database Management System (DBMS). Accumulations of enormous, extensive text based Information contain huge measure of organized data, which remains unstructured content. Important data is constantly hard to find in these documents. In this paper we proposed a novel approach that encourages the era of the organized metadata by recognizing records that are prone to contain data of investment and this data is going to be helpful for questioning the database. Here individuals will lying on your front to allot metadata identified with records which they transfer which will effortlessly help the clients in recovering the records.
\end{abstract}

Keywords: Collaborative Adaptive Data sharing platform (CADS), Annotation, metadata, structured information, queries.

\section{INTRODUCTION}

Many systems do not have the basic "attribute value" annotation that would make a querying feasible. Annotations that use "attribute value" pairs require users to be more principled in their annotation efforts. Users need to have good idea in using and applying the annotations or attributes. Even if the system allows users to annotate the data with such attribute-value pairs, the users are often unwilling to perform the task. Such difficulties results in very basic annotations that is often limited to simple keywords. Such simple annotations make the analysis and querying of the data cumbersome. Users are often limited to plain keyword searches, or have access to very basic annotation fields, such as "creation date" and "size of document".

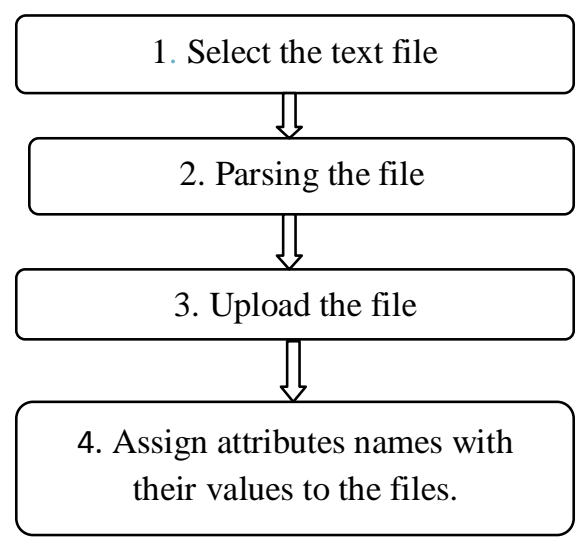

Fig.1 Information Extraction Algorithm
In this paper, we propose CADS (Collaborative Adaptive Data Sharing) platform which is an "annotate-as-youcreate" infrastructure that facilitates fielded data annotation. A key contribution of our system is the direct use of the query workload to direct the annotation process, in addition to examining the content of the document. Our aim is to prioritize the annotation of documents towards generating attribute names and attribute values for attributes that will often used by querying users and these attribute values will provide best possible results to the user wherein users will have to deal only with relevant result.

Our goal is to suggest annotations for a document.

1) Select a text file

2) Parse the text file. Ignore stop words from it and count frequency of high querying keywords which will be important for content based search. Maintain frequency count of thesekeywords appearing inonly single document.

3) Upload the file on to the server

4) Then fill all the annotations which are relevant to the document which can be useful for query based searching.

Example: year $=2012$, location='Nashik', author='Bill Gates' etc.

QV, CV Computation and Combining Algorithm:

1) Enter the queries for retrieving the document.

Example: location='Nashik' and year=2012.

2) Split the queries and pass it to database for retrieving.

3) Check all related results and show the related results to user. 
4) Formuch efficient and accurate result, user should try to enter maximum queries they can.

\section{CADS PRELIMINARY DESIGN}

The CADS system has two types of actors: producers and consumers. Producers upload data in the CADS system using interactive insertion forms and consumers search for relevant information using adaptive query forms. In the rest of the paper the term data usually refers to a document; other types of data are also possible, but we focus on documents for simplicity. Figure 1 presents a typical CADS workflow. Figure 2 shows the possible components of the two major CADS modules, the Insertion and Query modules.

(a)Insertion phase: The insertion phase begins with the submission of a new document to be included in the repository. After the user uploads the document, CADS analyzes the text and creates an adaptive insertion form with the set of the most probable <attribute name, attribute value) pairs to annotate the new document. The use fills this form with required information and submits it. The final stage consists of the storage of the associated document and metadata in the CADS repository. Going back to our disaster management motivating scenario, Figure 3 presents the adaptive insertion form for the hurricane advisory document. After the user submits the document, the system analyzes the content, and finds that the following attributes are relevant: "StormName", "StormCategory", "Warnings". These attributes are added toa set of default attributes like: "Document Type", "Date" and "Location", which are basic metadata that a domain expert has provided for an application. The "Description" attribute is used to input the whole text of the document.

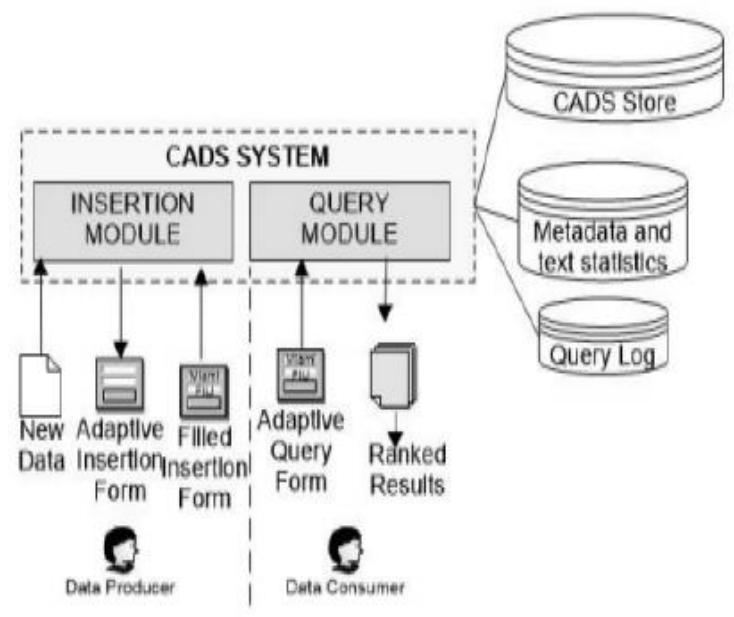

Fig 2: CADS Workflow

In addition to extracting attribute names, the adaptive insertion form also extracts the attribute values by employing IE algorithms. A confidence threshold for the IE must be set. A lower threshold may bias the user and lead to errors in the data, whereas a high threshold may lead to many empty textboxes, which may frustrate the user. Ideally, the erroneous valuesare corrected and the missing attribute values are manually inserted by the user. This means that the quality of the data depends on the reliability of the users. User trust and anti-spam techniques must beconsidered for large-scale deployments of CADS. As shown in Figure 3, attribute names and attributevalues are presented as text boxes. If the user wants to associate more than one value to an attribute - e.g.,multi-valued attributes like "Warnings"- then she can use the plus icon at the right to add attribute values. Each textbox has autocompletion capabilities, which exploit similar entries inserted before in the same attribute. It is also important, to notice that a user can add new attributes, which are not suggested by the adaptive form. The formprovides the option to do this task, in the spirit of the Google Base. When the user specifies a new attribute, CADS will try to match it to existing attributes and show to the user a few matching options. The user can reject these suggestions and go ahead adding the new attribute. In this way, advanced users can collaborate for the schema construction.

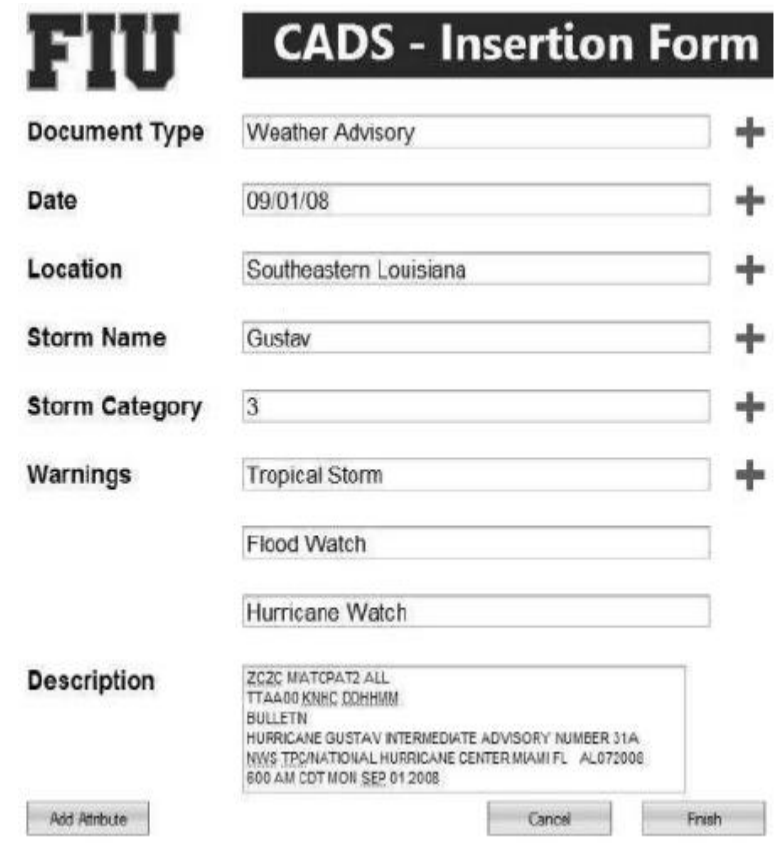

Fig3: Adaptive Insertion Form

(b) Query phase: In the query phase, the user ispresented with an adaptive query form (Figure 4), which supports 〈attribute name, attribute value)conditions. Initially, before CADS has began learning the information demand through processing the query workload, the query form only specifies the default attributes (e.g., "Document type", "Date","Location"). The user can specify additional 〈attribute name, attribute value〉 conditions. There is also a generic "Description" attribute where the user types keywords when she does not know how to put them in 〈attribute name, attribute value〉 conditions. The system discourages the user from just using the "Description" attribute, because this does not allow the system to learn 
the user information demand in a structured way, which in turn facilitates evolving the schema and performing schema mappings. In some cases the conditions may trigger additional attributes recommendation, which CADS believes could be helpful for the user to further refine the query. For instance, if the user specifies the attribute "Storm Category" and previous users who specified "Storm Category" also specified "Wind Speed", then the adaptive query form will suggest to the user the attribute "Wind Speed". Further, if the attribute specified by a user is similar to another existing attribute, CADS will suggest a mapping between the two attributes, in the spirit of pay-as-you-go integration. Also, the system may suggest replacing the text in the generic "Description" attribute value with some 〈attribute name, attribute value) conditions. When the user decides that her query form is complete, she submits the query. In this last phase CADS will find the most important pieces ofdata (e.g., document) for the query. The querying strategy must combine keyword search with uncertain structured query principles. The systemreturns a ranked list of the results, where the ranking is personalized. In order to personalize, CADS mayassume that users generally look for similar items every time they search. A user profile may also be used. Also, note that CADS will typically return whole documents in the result. However, if the schema of the repository is mature and the query is selective, it is possible to return specific attribute values, in a way similar to the NAGA system. The latter query result type is a possible future direction for CADS.

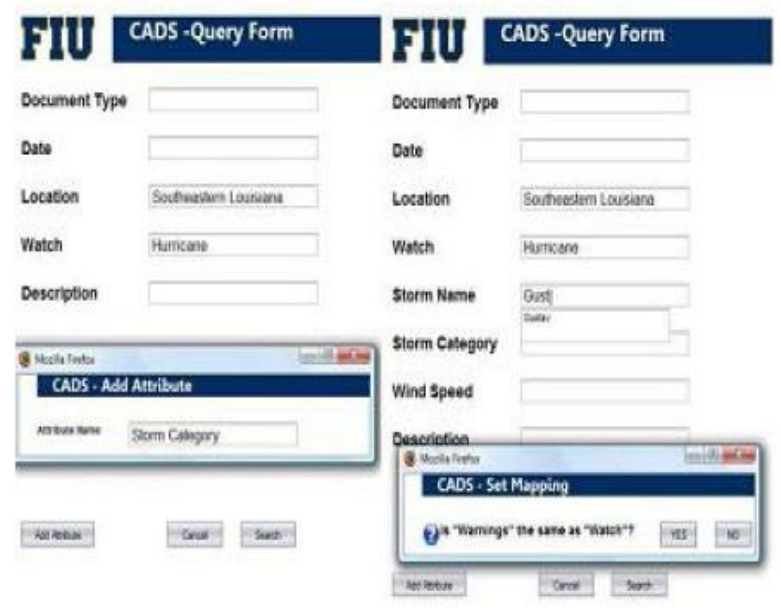

Fig 4: Adaptive Query Form.

In Figure 4 we show the progression of an adaptive query form in the disaster domain. In the left windowwe show the initial status of the query form. The generic form starts with some default attributes:"Document Type", "Location", "Description". The user is encouraged to specify other attributes, whichdo not only refine the query, but also help CADS learn the user information demand. For instance, inFigure 4 the user adds an attribute called "Storm Category" using the auxiliary window. Then,
theFormsuggests to the user to also include the attributes "Storm Name" and "Wind speed",which are correlated with "Storm Category" in the query workload.After that, the system tries to auto-compete the attributes value for "Storm Name" again using the past query workload.Finally", the system asks a pay as- you-go schema mapping question: if "Warnings isequivalent to "Watch", where the former is part of the existing schema (see Figure 4) and the latter is a user specified-attributes.

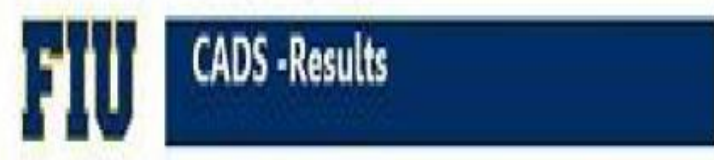

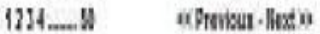 Inom

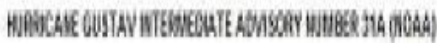

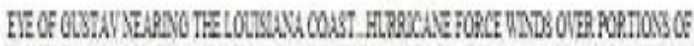

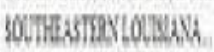

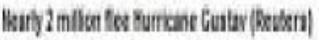

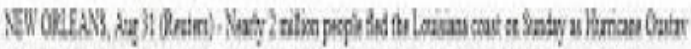

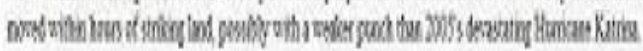

Fig 5: Query result

Figure 5 shows the results of the query. The document inserted in Figure 4 is the top result. Notethat each result in the list may partially or fully satisfy the query, and is owned by a user. The trust degree of the owner for the querying user may beused as one of the ranking factors, in addition to factors like relevance and importance.

\section{RELATED WORK}

Collaborative Annotation.

There are several systems that favour the collaborative annotation of objects and use previousannotations or tags to annotate new objects. There havebeen a significant amount of work in predicting the tags fordocuments or other resources (webpages, images, videos)[1], [2], [3], [4].Depending on the object and theuser involvement, this approaches, have different assumptionson what is expected as an input;nevertheless, the goals are similar as theyexpect to find missing tags that arerelated with the object. We argue that our approach isdifferent as we use the workload to augment the documentvisibility after the tagging process. Compared with the other approaches, precision is a secondary goal as weexpect that the annotator can improve the annotations onthe process. On the other hand, the discovered tags assist onthe tasks of retrieval instead of simply bookmarking. 


\section{Dataspacesand pay-as-you-go integration.}

The integration model of CADS is similar to that of Dataspaces [5], where a loosely integration model is proposed for heterogeneous sources. The basic difference is that Dataspaces integrate existingannotationsfor data sources, to answer queries. Our work suggests the appropriate annotation during insertion time, and also takes into consideration the query workload to identify the most promising attributes to add. Another related data model is that of Google Base [6], where users can specify their own attribute/value pairs, in addition to the ones proposed by the system. However, the proposed attributes in Google Base are hard-coded for each itemcategory (e.g., real-estate property). In CADS, the goal is to learn what attributes to suggest. Pay-as-you go integrationtechniques like PayGo [7] and [8] are useful to suggest candidate matching at query time. However, no previouswork considers this problem at insertion time, as in CADS. The work on Peer Data Management Systems [9] is aprecursor of the above projects.

\section{Query Form.}

To access database, forms-based query interfaces are used. The design of form based interfaces is a key step in the process of database. But in that interfaces, it is capable of expressing very limited number of queries. The form [10],[11], expresses all thequeries that an user may have. Form is a simple query interface used to access database. It doesn't require any additional knowledge from the part of user for processing forms. The users no need to worry about the internal language used or other types of internal organization. This query forms will give an overview of the underlying data. Most users will use these query forms to access database because of these simplicity. While creating form based interfaces, careful analysis is needed as any error will make the form ineffective. So creation of form must need full evaluation of the user preferences and user requirements. To design such forms, interface must develop a clear understanding about the data. A common problem of database systems is that it is very hard to give query for users who are uncomfortable with a formal query language. In order to solve this problem, form based interfaces are used. Here it wills automatically checks which questions are the most important for setting the query. One of the drawback of form based query interfaces is it is restrictive i.e.; it doesn't allow the user to express the query in another form.

\section{Information Extraction.}

Information Extraction [12] systems are used to extract information that is relevant to a particular document. Here the system focuses on different kinds of resources on the web: HTML tables and database behind forms. There are three types of systems: Text Runner system, web Tables, and deep web. The Text Runner system focus on unstructured data. The Web tables system extracts relational tables from HTML. The deep-web mainly focuses on backend database which are accessible via HTML forms.

\section{Document Annotations}

Normally, organizations generate and share their descriptionsof their services. Such data contain structured informationwhich buried under unstructured text. Information extractionalgorithms are expensive to process these unstructuredinformation, as sometimes these information does not focus onthe targeted information. A novel approach is present to findout the data which contain the information of interest. Thisinformation is used for querying the database. Here a joint utilization of the content and query workload is done.Collaborative Adaptive Data Sharing (CADS)[13],[14],[15] platform helps to annotate the documentas they created by theowner in addition to examining the full document later. Themain goal of this paper is to suggest annotations for thedocument. While identifying and suggesting attributes, theattributes must have high querying value with respect to thequery workload i.e., they must appear in many queries and alsohigh content value i.e., they must be relevant to the document.These suggested attributes were used for annotating thedocument.In this paper, users no need to examine the full document forsuggesting and identifying the attributes for annotating thedocument. The CADS will automatically suggest the neededattributes by checking the frequency and relevancy of eachattributes. Here attributes are generated by two processes inparallel i.e., by inspecting the content of the document and byinspecting the types of queries used. Normally attributevalueannotation is used. But it is limited to simple keywords.Querying from those annotations is very difficult as user haveto type more queries for accurate suggestions. Users must knowthe detailed schema and all field types to use. Another issuehere is it may sometimes have more attribute names for singleattribute.

\section{CONCLUSION}

The annotation system helps to reduce the query workload by automatically suggesting attributes. Many data mining techniques are proposed. Here some of the annotation schemes used is discussed. Also a brief description about the proposed methodology is given above. This will improve the searching process and also reduce workload and time. With the help of this technique, searching and analysis of document and webpage - metadata will become efficient and fast. Here attribute values are found that have frequent occurrence. Using these attribute values annotation process can be improved.

\section{REFERENCES}

[1] P. Heymann, D. Ramage, and H. Garcia-Molina, "Social Tag Prediction," Proc. 31st Ann. Int'1 ACM SIGIR Conf. Research and Development Information Retrieval(SIGIR'08),pp531-538

[2] Y. Song, Z. Zhuang, H. Li, Q. Zhao, J. Li, W.-C. Lee, and C.L. Giles, "Real-Time Automatic Tag Recommendation," Proc. 31st Ann. Int'l ACM SIGIR Conf. Research and Development in Information Retrieval(SIGIR '08), pp. 515-522, http://doi.acm.org/10.1145/1390334.1390423, 2008. 
[3] D. Eck, P. Lamere, T. Bertin-Mahieux, and S. Green, "Automatic Generation of Social Tags for Music Recommendation," Proc .Advances in Neural Information Processing Systems 20, 2008.

[4] B. Russell, A. Torralba, K. Murphy, and W. Freeman, "LabelMe: A Database and Web-Based Tool for Image Annotation," Int'1 J. $\begin{array}{lllll}\text { Computer Vision, } & \text { vol. } & 77, & \text { pp. } & 157\end{array}$ 173,http://dx.doi.org/10.1007/s11263-00700908,2008,doi,10.1007/s11263-007-0090-8.

[5] M. Franklin, A. Halevy, and D. Maier, "From Databases to Dataspaces: A New Abstraction for Information Management", SIGMOD Record, vol. 3 pp.2734. http//doi.acm.org.10.1145/1107499.1107502,Dec.2005.

[6] "Google",GoogleBase,http://www.google.com/base',2011.

[7] J. Madhavan et al., "Web-Scale Data Integration You Can Only Afford to Pay as You Go," Proc. Third Biennial Conf. Innovative Data Systems Research (CIDR), 2007.

[8] S.R. Jeffery, M.J. Franklin, and A.Y. Halevy, "Pay-as You-Go User Feedback for Dataspace Systems," Proc. AC SIGMOD Int'l Conf. Management Data, 2008.

[9] Halevy, Z. Ives, D. Suciu, and Tatarinov, "Schema Mediation in Peer Data Management Systems," Proc. 19th Int'l Conf. Data Eng., pp. 505-516, Mar. 2003

[10] M. Jayapandian and H.V. Jagadish, "Automated Creation of a Forms-Based Database Query Interface,” Proc. VLDB Endowment, vol.1, pp. 695-709, http://dx.doi.org/10.1145/ 1453856.1453932, Aug. 2008

[11] M. Jayapandian and H. Jagadish, "Expressive Query Specification through Form Customization," Proc. 11th Int'l Conf. Extending Database Technology: Advances in Database Technology (EDBT '08),pp. 416-427, http://doi.acm.org /10.1145/1353343.1353395, 2008.

[12] M.J. Cafarella, J. Madhavan, and A. Halevy, "Web-Scale Extraction of Structured Data," SIGMOD Record, vol. 37, pp. 5561,http://doi.acm.org/10.1145/1519103.1519112, Mar. 2009.

[13] Eduardo J. Ruiz, Vagelis Hristidis and Panagiotis G. Ipeirotis "Facilitating Documents Annotation Using Content and Querying Value" IEEE transaction on Knowledge and Data Engineering, Vol 26, N0.2, February 2014.

[14] M.Venkateswarlu, and N.Siddaiah "A Probabilistic Framework to Annotate Document Based On Document Content And Query Workload" ,Proc .International Journal Of Research In Advanced Technologies, vol 4. July.2015.

[15] N.S.Sabna and S. Jayalekshmi “A Review On Annotation Process ",Proc. International Journal Of Engineering And Computer Science-Vol.4, July 2015, Page No. 13264-13267 